\title{
Preface to the special issue dedicated to Professors Eiichi Bannai and Hikoe Enomoto on their 75th birthdays
}

\author{
Jack Koolen ${ }^{1}$ - Akihiro Munemasa ${ }^{2}$ - Atsuhiro Nakamoto ${ }^{3} \cdot$ Katsuhiro Ota $^{4}$. \\ Akira Saito ${ }^{5}$
}

Published online: 8 October 2021

(c) The Author(s), under exclusive licence to Springer Japan KK, part of Springer Nature 2021
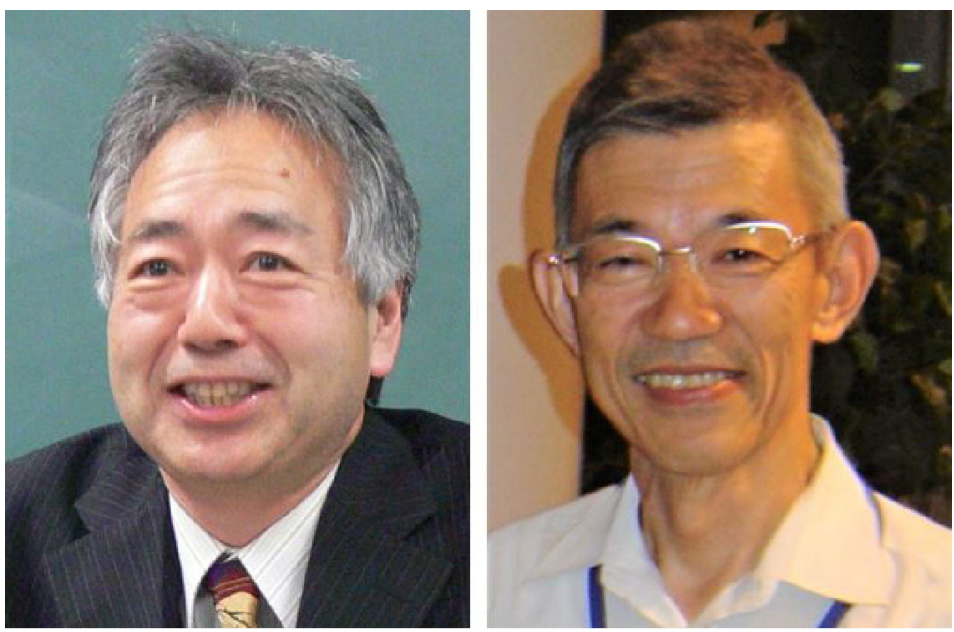

Professor Eiichi Bannai (left) and Professor Hikoe Enomoto

Bannai and Enomoto are leading pioneers of combinatorics in Japan. When they were students, combinatorics was not a popular area of mathematics. They entered the graduate school of Department of Mathematics, The University of Tokyo in 1968 and they started studying algebra under the same supervisor, Professor Nagayoshi Iwahori. But for some reason, both of them were attracted by combinatorics, and their research interest shifted. Bannai selected a research area in algebraic combinatorics. He built up the theory of association schemes by producing a number of deep results. Enomoto worked in a broad spectrum of combinatorics, especially graph theory. One of his chief achievements was answering Vašek Chvátal's conjecture that every $k$-tough graph $G$ with $|V(G)|$ even contains a $k$-factor. Bannai has been a member of the editorial board

This issue is dedicated in honor of Professors Eiichi Bannai and Hikoe Enomoto on the occasion of their 75 th birthdays.

Extended author information available on the last page of the article 
from the first issue, while Enomoto was managing editor of Graphs and Combinatorics during 1993-1999.

Eiichi Bannai moved to The Ohio State University and later returned to Japan at Kyushu University. Hikoe Enomoto worked at many universities in Japan, including The University of Tokyo, Keio University and Hiroshima University. They actively worked in combinatorics and guided many graduate students. By their research and education, combinatorics flourished in Japan. Many people earned Ph.D under the supervision of Bannai and Enomoto. These second generation has actively been doing research and bringing up the third generation. We would say that more than half of Japanese combinatorialists have a connection with Bannai or Enomoto in academic genealogy. Combinatorics is now a well-established area in the Japanese mathematical community.

Bannai and Enomoto also made a number of collaborative work with mathematicians outside Japan. Their friendly and outgoing characters charmed the collaborators, and many of them have kept a firm friendship with them. When we started the project of this special issue, many of these friends, together with their academic descendants, happily helped us and submitted papers. We hope that these papers will academically celebrate the birthdays of Bannai and Enomoto.

Happy 75th birthday, Eiichi and Hikoe!

Publisher's Note Springer Nature remains neutral with regard to jurisdictional claims in published maps and institutional affiliations.

\section{Authors and Affiliations}

\section{Jack Koolen ${ }^{1} \cdot$ Akihiro Munemasa $^{2}$ - Atsuhiro Nakamoto ${ }^{3} \cdot$ Katsuhiro Ota ${ }^{4}$. Akira Saito ${ }^{5}$}

$凶$ Akira Saito

asaito@chs.nihon-u.ac.jp

1 School of Mathematical Sciences, University of Science and Technology of China (USTC), Hefei, Anhui, China

2 Division of Mathematics, Graduate School of Information Sciences, Tohoku University, Sendai, Japan

3 Division of Social Environment and Information, Faculty of Environment and Information Sciences, Yokohama National University, Yokohama, Japan

4 Department of Mathematics, Faculty of Science and Technology, Keio University, Yokohama, Japan

5 Department of Information Science, Nihon University, Tokyo, Japan 